\title{
On the Origin of the Apparent Volume of Distribution and Its Significance in Pharmacokinetics
}

\author{
Michalakis Savva \\ South University, Savannah, USA \\ Email: msavva@southuniversity.edu,micsavva@gmail.com
}

How to cite this paper: Savva, M. (2022) On the Origin of the Apparent Volume of Distribution and Its Significance in Pharmacokinetics. Journal of Biosciences and Medicines, 10, 78-98.

https://doi.org/10.4236/jbm.2022.101008

Received: December 17, 2021

Accepted: January 21, 2022

Published: January 24, 2022

Copyright (c) 2022 by author(s) and Scientific Research Publishing Inc. This work is licensed under the Creative Commons Attribution International License (CC BY 4.0)

http://creativecommons.org/licenses/by/4.0/

\begin{abstract}
The apparent volume of distribution was defined for the first time as the phase volume that can hold the total amount of a substance at the measured phase substance concentration, in a system composed of two immiscible media that are in contact under conditions of constant phase volumes, at equilibrium. Its value is not affected by the total system solute mass and it only depends on the total system volume, the phase volumes and the affinity of the solute for the two phases in the system. Using this new concept of the apparent volume of distribution, we were able to demonstrate that under certain conditions compartment volumes in multi-compartment and multi-phasic pharmacokinetic models represent the actual physiological volumes of body fluids accessible by drugs. The classical pharmacokinetic models are now fully explained and can be used to provide accurate estimation of the pharmacokinetic parameters for hydrophilic drugs. In contrast, in the absence of tissue-plasma partition coefficients, lipophilic drugs that do not follow a onecompartment model are unlikely to be adequately described with classical multi-compartment pharmacokinetic models.
\end{abstract}

\section{Keywords}

Apparent Volume of Distribution, Partition Coefficient, Phase Extraction, Pharmacokinetic Compartmental Modeling, Physiologically-Based Pharmacokinetic Modeling

\section{Introduction}

In 1934, Dominguez defined the volume of distribution for the first time as the volume of body fluid dissolving nonmetabolizable excretable substances at the same concentration as that in plasma under the assumption that equilibrium is 
maintained during the exponential decrease of excretion [1] [2]. He also stated that the volume of distribution is proportional to the sum of its rate of utilization and excretion (now known as Clearance) and inversely proportional to the elimination rate constant from the body. Still, eighty-four years later, hundreds of articles define the (apparent) volume of distribution of a drug as a mathematical ratio, a proportionality constant, a fudge factor, a hypothetical, theoretical, ideal, imaginary or apparent volume that has no direct anatomical or physiologic meaning, but somehow it relates the amount of drug in the body to the plasma drug concentration.

It would be interesting to know that Dominguez never used the term apparent or hypothetical volume of distribution. In his two articles, the calculated volumes of distribution for creatinine and xylose were within the normal parameters of the total volume of body fluids [1] [3]. However, over the years there was an increasing number of investigators that were calculating a variable volume of distribution for many excretable compounds, much higher than the total body water. The volume of distribution of a substance was usually determined by the difference of a dose given systemically and the amount excreted in the urine divided by the serum drug concentration. The first article that described the term apparent volume of distribution appeared four years after the pioneering work of Dominguez [4]. According to these investigators, the apparent volume of distribution would differ from the true volume of distribution if the substance of interest is somehow produced endogenously or subjected to metabolism, is not uniformly distributed in body fluids or is not solely excreted in the urine. One could also note that they didn't think that the apparent volume of distribution of a substance should have a constant value. More bothersome to them was not the observed variability but rather the lack of a trend into the variability of the apparent volume of distribution with time.

Can this abstracted concept of the (apparent) volume of distribution of a substance be recreated and studied in an isolated system? Dominguez's statements are a description of the physical significance of a hydrophilic drug's volume of distribution and its relationship to other important physiological parameters but they certainly do not constitute a technical definition of the apparent volume of distribution of a substance. The origin of a substance's apparent volume of distribution lies in the substance distribution between two immiscible solvents that are in contact in a closed system. The affinity of a substance for the two phases is denoted by the value of the partition or distribution coefficient $\mathrm{K}$, which is calculated from the ratio of the solute concentration in the two phases and is considered to be a macroscopic thermodynamic parameter that bears a constant value at constant temperature and pressure [5]. A scientific definition of the apparent volume of distribution will certainly reject or validate and expand its current use in the field of pharmacokinetics. For once, it will become apparent that the apparent volume of distribution of a drug in multi-compartment pharmacokinetic models is related to its actual volume of distribution in the body. 


\section{Methods}

The apparent volume of distribution was studied in a series of virtual distribution experiments of a solute in a biphasic system of constant composition, at constant temperature (Figure 1 ). The affinity of the solute for phase 2 was set to be three times that of phase 1 (partition coefficient, $K_{2,1}=3$ ), in all experiments. At equilibrium, solute concentration in the two phases, $C_{1}$ and $C_{2}$, was calculated using $K_{2,1}=\frac{C_{2}}{C_{1}}$ and a mass balance equation.

\section{Results}

Adding the solute into pure solvent 1 or solvent 2 results in a solute volume of distribution equaling the volume of the closed system (Equation (1); Figure 1, left). When a solute is added to a solvent, initially the system may be seen as a myriad kinetically different compartments, as the distribution of solute is dictated by its diffusivity in the solvent. This is however irrelevant, as the volume of distribution of a substance is an equilibrium property of the system and at equilibrium all these kinetically different phases will merge to form a single true solution.

$$
V_{d}=V_{s}=\frac{x_{s}}{C_{s}}
$$

As with the partition coefficient, there are as many volumes of distribution and as many apparent volumes of distribution as the number of phases in a system. The volume of distribution of a substance in a phase is the volume of that phase in the system. On the other hand, the apparent volume of distribution of a solute associated with the solvents of a two-phase system (Figure 1, middle panel) can be determined at equilibrium using a modified mass balance equation (Equation (2)).

$$
\begin{gathered}
V_{d, 1} \cdot C_{1}=V_{1} \cdot C_{1}+V_{2} \cdot C_{2} \\
V_{d, 1}=V_{1}+V_{2} \cdot K_{2,1} \\
K_{2,1}=\frac{V_{d, 1}}{V_{d, 2}} \\
V_{d, 1}=\frac{x_{s}}{C_{1}}
\end{gathered}
$$
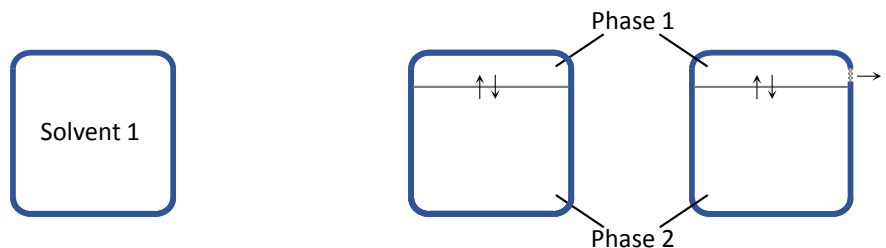

Figure 1. Left: Solute added in a closed compartment containing only solvent 1 or solvent 2. Middle: Solute added in a biphasic closed system of immiscible solvents 1 and 2. Right: Solute partitions into the two phases and is allowed to escape out of the open system from phase 1. 


$$
\begin{gathered}
V_{d, 2}=\frac{x_{s}}{C_{2}} \\
\frac{V_{1}}{V_{d, 1}}=\frac{x_{1}}{x_{s}} \\
\frac{V_{d, 2}}{V_{2}}=\frac{x_{s}}{x_{2}}
\end{gathered}
$$

where $x_{1}, x_{2}, x_{s}, V_{1}, V_{2}, V_{s}, V_{d, 1}$ and $V_{d, 2}$ are the mass of solute in phase 1, solute mass in phase 2 , the total solute mass in the system, the volume of phase 1 , the volume of phase 2 , the total volume of the system, and the substance's apparent volume of distribution associated with phase 1 and phase 2 , respectively.

Clearly, the apparent volume of distribution of a solute in a phase is independent of the mass of the solute in the system and it only depends on the distribution coefficient and the volumes of the two immiscible media that are in contact. The higher the partition coefficient of a solute for a phase the smaller is the value of the solute's apparent volume of distribution for that phase. Secondly, the apparent volume of distribution associated with a solute in a phase can be defined as the volume of that phase that can form a solution with the total mass of the solute in the system at a specified phase solute concentration. Adding for example, $60 \mathrm{mg}$ of solute into a system composed of $3 \mathrm{~L}$ of phase 1 and $39 \mathrm{~L}$ of phase 2 with a partition coefficient $K_{2,1}=3$, results in $V_{d, 1}=120 \mathrm{~L}, V_{d, 2}=40 \mathrm{~L}$, $C_{1}=0.5 \mathrm{mg} / \mathrm{L}$, and $C_{2}=1.5 \mathrm{mg} / \mathrm{L}$. Accordingly, in order to accommodate 60 $\mathrm{mg}$ of the solute in phase 1 without causing any change in the original equilibrium solute concentration, you will have to add $\frac{V_{d, 1}}{V_{1}}$ times bigger mass of solute $(2400 \mathrm{mg})$ into a system composed of $\frac{V_{d, 1}}{V_{1}}$ times larger phase volumes, that is, $120 \mathrm{~L}$ of phase 1 in contact with $1560 \mathrm{~L}$ of phase 2 . Changing the volume of the two phases will obviously create a new apparent volume of distribution $V_{d, 1}^{\prime}=4800 \mathrm{~L}$. We can easily verify using Equation (4) that with $V_{d, 1}^{\prime}=4800 \mathrm{~L}$ and the total mass of solute added in the system $x_{s}=2400 \mathrm{mg}, C_{1}$ stays constant at $0.5 \mathrm{mg} / \mathrm{L}$.

The first simulations were conducted to investigate the effect of varying the mass of solute in the system on the solute's apparent volume of distribution. As is shown by the equilibrium line in the distribution isotherms of Figure 2, increasing the mass of solute in the system at constant phase volumes, results in increased equilibrium solute concentration in both phases but the ratio of the two concentrations is always equal to the value of the partition coefficient. The increase in solute concentration in the two phases with increasing solute added into the system is linear and the inverse slope is equal to the apparent volume of distribution of the solute in each phase (Equations (4)-(5)). Apparently, the increase in the mass of solute in the system is accompanied by an exact proportionate increase of the mass of the solute in each phase and as a result, the apparent 

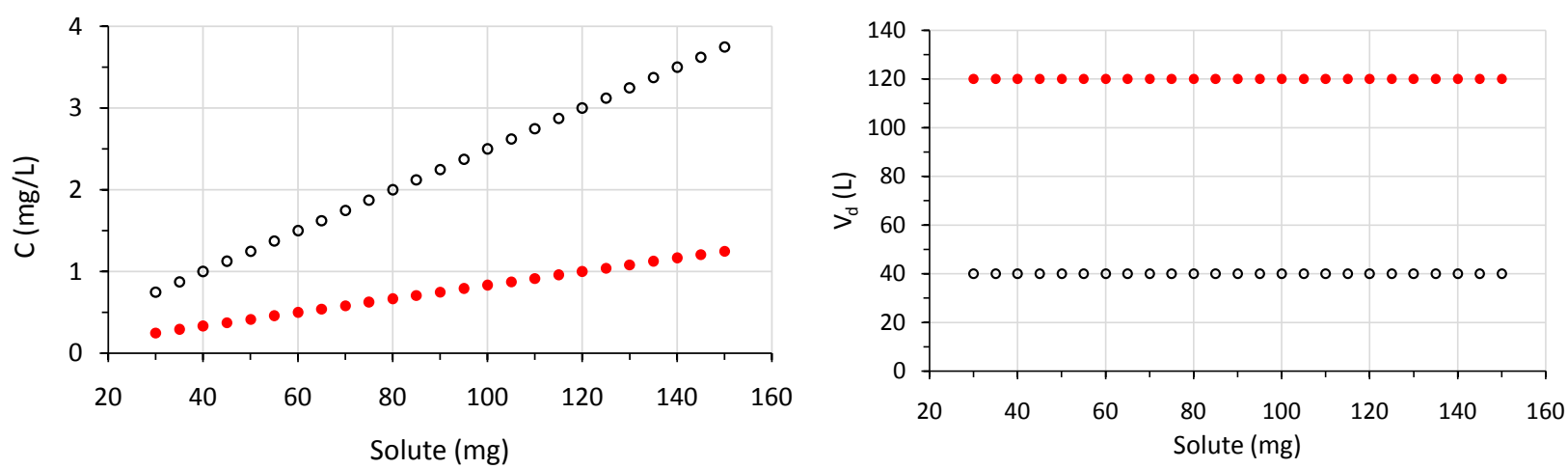

Figure 2. Solute concentration (Left) and solute apparent volume of distribution (Right) in phase 1 (solid circles; $\left.C_{1}, V_{d, 1}\right)$ and in phase 2 (empty symbols; $C_{2}, V_{d, 2}$ ). The mass of the solute in the system (Figure 1, middle) was varied from 30 to $150 \mathrm{mg}$ while the distribution coefficient and the volumes of the two phases were kept constant in all experiments, $K_{2,1}=3, V_{1}=3 \mathrm{~L}$, $V_{2}=39 \mathrm{~L} \quad\left(V_{s}=42 \mathrm{~L}\right)$.

volumes of distribution of the solute associated with the two phases, $V_{d, 1}$ and $V_{d, 2}$, stay constant. The ratio of the two apparent volumes of distribution equals the value of the partition coefficient (Figure 2, right, and Equation (3)).

We continued the sensitivity analysis of our model with the effect of system volume changes on the solute apparent volumes of distribution. At constant partition coefficient and mass of the solute in the system, we have examined three different scenarios where changes in the total volume of the system were all absorbed by phase 1, phase 2 or by both phases at equal proportions. At increasing $V_{2}$ and constant $V_{1}$, decreased distribution of the solute in phase 1 at constant mass of solute in the system causes a reduction in $C_{1}$ which in accord with Equation (4b), brings about a pronounced increase in $V_{d, 1}$, (Figure 3, top panels, solid triangles). The mass of solute in phase 2 increases at a rate slower than the corresponding increase in the phase volume (Equation (5b)). The concentration of the solute in phase 2, $C_{2}$, is therefore decreasing with phase volume increase and as a result, $V_{d, 2}$ experiences the same drastic increase as $V_{d, 1}$ (Figure 3, top panels, empty triangles). The trend at constant $V_{2}$ and increasing $V_{1}$ on the apparent volume of distribution is similar but the effects are less pronounced due to a relatively bigger initial $V_{2}$ and the preferential partitioning of the solute in phase 2 (Figure 3, top panels, solid and empty squares).

$$
\begin{gathered}
C_{1}=\frac{x_{s}}{V_{1}+K_{2,1} \cdot V_{2}} \\
x_{2}=\frac{x_{s}}{\frac{V_{1}}{K_{2,1} \cdot V_{2}}+1}
\end{gathered}
$$

As anticipated from Equations (4b) and (5b), increasing the total system volume at constant phase volume ratio has no effect on the mass of solute partitioned in each of these phases. As a result, both $C_{1}$ and $C_{2}$ decrease with increased $V_{1}$ and $V_{2}$ and consequentially the apparent volumes of distribution 

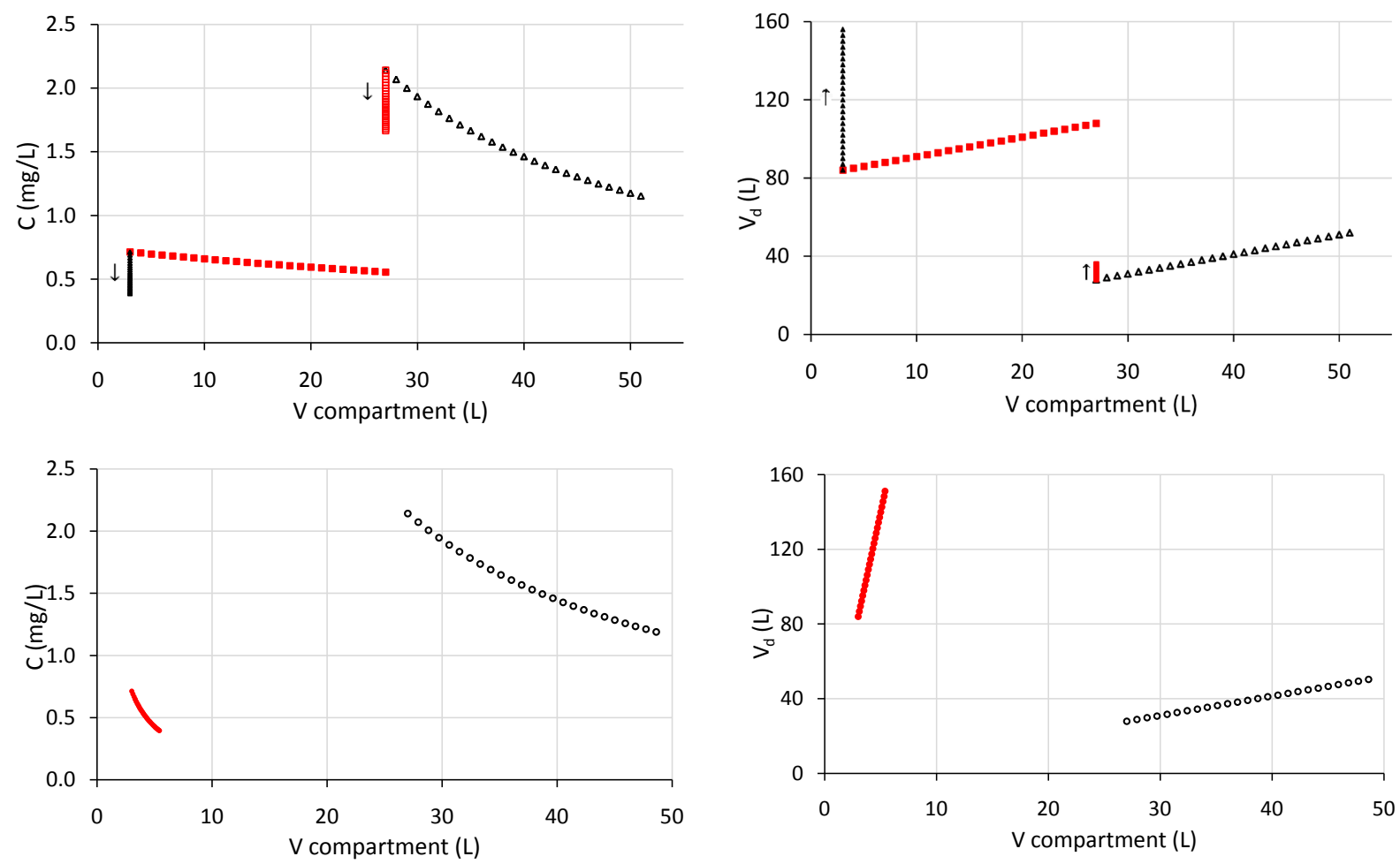

Figure 3. Solute concentration (Left) and solute apparent volume of distribution (Right) in phase 1 (solid symbols; $C_{1}, V_{d, 1}$ ) and in phase 2 (empty symbols; $C_{2}, V_{d, 2}$ ). All simulations were conducted with $K_{2,1}=3$. The mass of the solute in the system was kept constant while the volume of the system $\left(V_{s}\right)$ was varied from $30 \mathrm{~L}$ to $54 \mathrm{~L}$. Solid and empty triangles: Phase 1 was kept constant, $V_{1}=3 \mathrm{~L}$, while the volume of phase 2 was varied, $27 \mathrm{~L} \leq V_{2} \leq 51 \mathrm{~L}$. Solid and empty squares: Phase 2 was kept constant, $V_{2}=27 \mathrm{~L}$, while phase 1 was varied, $3 \mathrm{~L} \leq V_{1} \leq 27 \mathrm{~L}$. Arrows indicate parameter change direction with increasing system volume, at corresponding constant compartment volume (phase 1 or phase 2). Solid and empty circles: increasing $V_{s}$ at constant phase volume ratio $\frac{V_{2}}{V_{1}}=9 ; 3 \mathrm{~L} \leq V_{1} \leq 5.4 \mathrm{~L}, 27 \mathrm{~L} \leq V_{2} \leq 48.6 \mathrm{~L}$.

of both phases, $V_{d, 1}$ and $V_{d, 2}$, increase as well (Figure 3, bottom panels, solid and empty circles). Evidently, an increase in the volume of the system will always result in an increase of the solute apparent volume of distribution associated with both compartments, but the normalized apparent volume of distribution per phase volume increase is constant.

Our last simulation carried out at constant $x_{s}$ and $V_{s}$ indicated that, $V_{d, 1}$ decreases with increasing $V_{1}$ and decreasing $V_{2}$ whereas $V_{d, 2}$ increases with decreasing $V_{1}$ and increasing $V_{2}$ (Figure 4). The changes in the values of the apparent volume of distribution are governed by the different affinity of the solute for the two phases and they are proportional to the phase volume changes.

\section{Discussion}

\subsection{The Apparent Volume of Distribution}

This work was initiated as a thought experiment in an attempt to explain pharmacokinetic compartment volumes. We set out to look for a property, that relates 

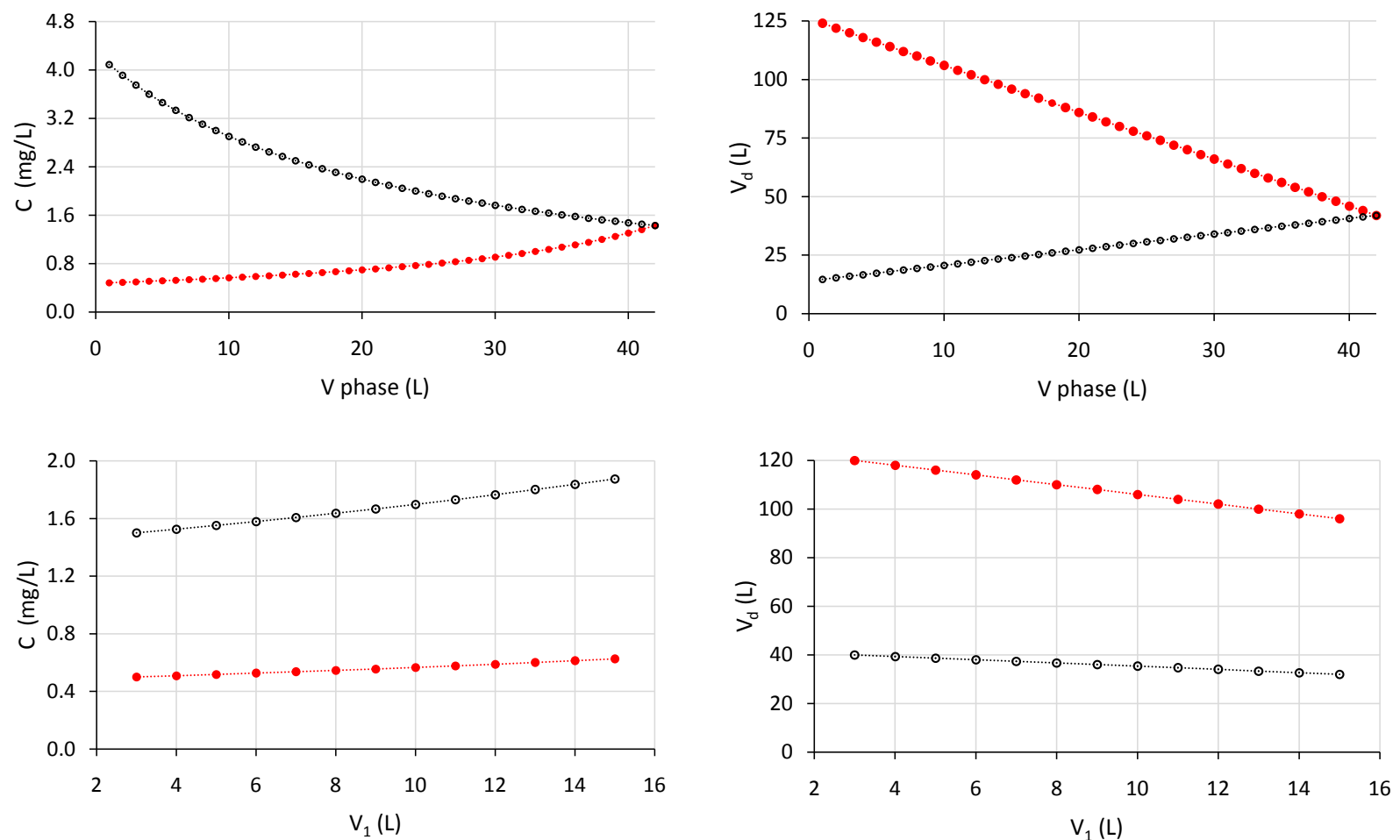

Figure 4. Solute concentration (Left) and solute apparent volume of distribution (Right) in phase 1 (solid symbols; $C_{1}$, $V_{d, 1}$ ) and in phase 2 (empty symbols; $C_{2}, V_{d, 2}$ ). All simulations were conducted with the partition coefficient, the mass of the solute and the volume of the system being kept constant at $3,60 \mathrm{mg}$ and $42 \mathrm{~L}$, respectively. $V_{1}$ was increased at the expense of $V_{2}$, and vice versa, at constant volume system. Top: $0 \leq V_{1} \leq 42 \mathrm{~L}, 42 \mathrm{~L} \geq V_{2} \geq 0 \mathrm{~L}$. Bottom: $3 \mathrm{~L} \leq V_{1} \leq 15 \mathrm{~L}, 39 \mathrm{~L} \geq V_{2} \geq 27 \mathrm{~L}$.

the total mass of a substance in a closed system composed of two immiscible solvents to its concentration in one of the phases and stays constant regardless of the total mass of the solute, as in a clinical setting we are routinely measuring plasma concentration but seeking information about drug distribution in other body tissues. As this system will always remain separated, we used a modified mass preservation equation (Equation (2)) where the final equilibrium state is expressed in terms of the substance equilibrium concentration and volume in one of the phases, instead of the final mixture concentration and final volume that we routinely use during solution formation by mixing two or more solutions [5] [6]. Notice that the product $V_{d, 1} \cdot C_{1}$ must also equal the total mass of substance in the system, $x_{s}$. We designated the solute's apparent volume of distribution as the phase volume that yields the total solute mass in the system when it is multiplied with the corresponding phase solute concentration and proceeded to study its properties in virtual distribution experiments.

The apparent volume of distribution is a physical property that only depends on the type of matter. It is related to a substance distribution into two immiscible solvents of constant volume and can be defined as the volume of a phase that can contain the total mass of a substance added into the system while maintaining the phase substance concentration constant. Each phase has its own solute 
apparent volume of distribution. One of the areas that the apparent volume of distribution has direct application is solvent extraction techniques [5]. The efficiency of an extraction method is evaluated by the fraction of the solute that moves into the extracting phase (Equation (4a)). Using the concept of the apparent volume of distribution the extraction efficiency can be calculated from a single concentration measurement and the known volume of the assayed phase. Alternatively, the capacity of a solvent to extract a substance can be evaluated by addition of increasing amounts of substance in the system. The apparent volume of distribution can be calculated from the slope of linear regression analysis of measured phase solute concentrations as a function of solute mass in the system (Figure 2, left) and can be used to optimize the product load in a solvent system in order to achieve a certain concentration of the substance in the extracting phase [5].

In compartmental modeling, each compartment has its own solute's apparent volume of distribution only if the compartments constitute different phases. Compartments that differ kinetically with regards to solute distribution but are otherwise compositionally similar have the same apparent volume of distribution. As per our analysis (Figure 1, left), the absence of different phases in such a system automatically renders the solute apparent volume of distribution to be equal to the total volume of the kinetically different compartments. The ratio of the solute concentration in two phases at equilibrium is defined by the affinity of the solute for each phase and equals the value of the partition coefficient regardless of the solute mass and volume of the two phases in the system. As with the partition coefficient, there are as many solute apparent volumes of distribution as the number of phases in a system. However, unlike the partition coefficient, the value of the apparent volume of distribution of a substance is dependent on the actual phase volumes. The ratio of the solute concentration maybe dictated exclusively by the value of the partition coefficient but the actual solute concentration in the two phases is also controlled by the real phase volumes, which in turn, they also control the value of the apparent volume of distribution. At constant phase volumes, phase solute concentrations are directly proportional to the total mass of the solute in the system. This is why the apparent volume of distribution is such an important property in pharmacokinetic compartment models. From its magnitude, the dose administered and its plasma concentration, one can calculate exactly how much drug is in each compartment, at a state of equilibrium. In addition, an apparent volume of distribution larger than the volume of all the compartments together that are of unknown composition and unknown volume, suggests that the drug has a higher affinity toward the peripheral compartments and not for the assayed central compartment [5].

In vivo, the apparent volume of distribution of a drug can be defined as the volume of the assayed phase that can contain a drug dose at the specified measured drug concentration in the assayed phase. We must keep in mind that although drug concentrations are usually measured in plasma, the apparent vo- 
lume of distribution of a drug doesn't have to relate only to the assayed plasma but rather to the whole phase or compartment that the plasma is kinetically part of, including other extracellular fluids. Therefore, changes in drug dose are reflected in changes of drug concentration in the assayed phase (Equation (4b)). It is on this basis that drug doses can be correlated with measurements of drug concentration in body fluids to accurately assess the apparent volume of distribution of a drug with respect to the assayed phase. Single ascending dose studies can be employed to demonstrate presence of linear pharmacokinetics and as we have shown in Figure 2, they can also be used to determine the drug's apparent volume of distribution. The advantage of this method is that $V_{d}$ is determined from multiple dose-concentration measurements regardless of compartment model [5] [7].

\subsection{The One-Compartment Model}

The one-compartment pharmacokinetic model is an open model that assumes instantaneous distribution of the drug from the central circulation to all other tissues in the body. As we have already seen, the apparent volume of distribution of a substance in a system composed of a single phase must be equal to the compartment volume (Equation (1)). Then why the apparent volume of distribution of most lipophilic drugs that their disposition follows a one-compartment model is larger than the volume of total body fluid? Is the one-compartment model a single phase? Although it lumps all tissues that display similar rates of drug uptake together in a single compartment, it is wrong to assume that the compartment is uniform. In fact, the one-compartment pharmacokinetic model recognizes that plasma drug concentration is most commonly some multiple of the drug concentration in other tissues. It acknowledges and feels the presence of other phases via the changes in the pharmacokinetic parameters that are calculated from concentration measured in the single compartment but it is not designed to see any of those phases that are maybe in contact with the compartment. So, although it calculates correctly the $V_{d}$ of a drug from its initial plasma drug concentration after an IV bolus dose, it fails to gather information about the actual volume of the assayed phase except in few distinct cases. Current literature claims that drugs with an apparent volume of distribution about $40 \mathrm{~L}$ or $60 \%$ of total body weight in healthy adults, have access to the total body fluid. Using Equation (2), an average $V_{\text {extracellular }}=15 \mathrm{~L}$ and $V_{\text {intracellular }}=27 \mathrm{~L}$, we find that the only way to get a drug apparent volume of distribution equal to the total volume of body fluids is when the drug partitions in extracellular and intracellular fluids with an equal affinity $\left(K_{2,1}=1\right)$. We can also say with certainty that drugs with a $3 \mathrm{~L}<V_{d} \leq 15 \mathrm{~L}$ are hydrophilic compounds that may diffuse through the capillary endothelium but are otherwise unable to transverse plasma cell membranes into cells. These drugs do not have to follow a one-compartment model if their distribution into the various extracellular fluids is kinetically different but an apparent volume of distribution in that range suggests the 
presence of only a single phase. Drugs with an apparent volume of distribution about $3 \mathrm{~L}$ follow compositionally and kinetically the one-compartment model. Apparently, these molecules do not have any affinity for any tissue other than the plasma or their size is prohibitively large to escape into the extravascular environment via the capillaries.

Lipophilic drugs are known to have apparent volumes of distribution much larger than the total body fluid leaving little drug in the plasma. Some of them can be simulated using an open one-compartment pharmacokinetic model. Apparently, distribution of the drug to extracellular fluids (phase a) and other tissues (phase $b$ ) is taking place at the same rate, and hence, drug disposition can be modeled using a single bi-phasic compartment. The second phase is the tissues that have a high affinity for the drug. The product of the tissue-plasma partition coefficient and the tissue phase volume explains why the $V_{d, a}$ of these drugs can be much higher than total body fluid volume. Therefore, even in the absence of any information about the high drug affinity tissues, Equation (6) can still be used to calculate the drug's $V_{d, a}$ from measured plasma drug concentration, $C_{a}$.

$$
\left(V_{a}+V_{b} \cdot K_{b, a}\right)=\frac{x_{s}}{C_{a}}
$$

There is one very important detail about the open one-compartment pharmacokinetic model that we must always remember. The instantaneous distribution of the drug to all tissues during drug administration implies instantaneous redistribution of the drug from the surrounding tissues to the intravascular volume at periods of no drug administration where only the process of drug elimination is taking place. Therefore, in the one-compartment open model, there is a continuous distribution equilibrium of the substance at all times. This statement has such profound consequences now that we have defined the apparent volume of distribution. The difficulty in determining the apparent volume of distribution is to know the amount of drug in the body at the time of concentration measurement. One of the most common ways to determine the apparent volume of distribution after an IV bolus injection is at zero time where the drug amount is equal to the dose. Once the $V_{d}$ of a drug is determined, Equation (4) or Equation (6) can be used to calculate the amount of drug in the body at all times because the open one-compartment pharmacokinetic model assumes a state of permanent substance distribution/redistribution equilibrium between the vascular, extravascular and intracellular fluids. We don't need to know the elimination rate constant nor the clearance to calculate a dose aimed to achieve a certain plasma drug concentration or to calculate the amount of drug in the body from measured drug plasma concentrations.

Equation (6) (or Equation (4)) is another version of the clinically useful loading dose equation that can be used to calculate booster doses in order to quickly raise the plasma drug concentration to the desired levels within therapeutic range in the plasma, strictly in the one-compartment model. This equation can- 
not be used in multi-compartment models as in those models, solute distribution equilibrium is not instantaneous and therefore it is not the dose that is related to the plasma drug concentration but the amount of drug remaining in the body at the time where the momentary distribution equilibrium is observed. Notice that we have two issues in multi-compartment models: first, the apparent volume of distribution cannot relate an intravenous bolus injection with plasma drug concentration at zero time and second, the apparent volume of distribution can relate the amount of drug in the body with the plasma drug concentration only at the times of momentary substance distribution equilibrium between compartments. The only exception to this rule is when the drug is administered by a slow constant rate-slower than the rate of drug distribution to the tissues-continuous intravenous infusion. In this case, at steady state there is essentially no fluctuation of the drug concentration in the plasma and regardless of compartment model, Equation (4) can used to calculate loading or booster doses to achieve desired steady state plasma or central phase drug concentrations. The apparent volume of distribution at steady state conditions during constant rate continuous IV drug infusion can be calculated using Equation (7).

$$
V_{d, 1}=\frac{k_{0} \cdot t_{1 / 2}}{\ln 2 \cdot C_{1, a v e, s s}}
$$

The rate of drug administration $k_{0}$ is in units of mass per time, $t_{1 / 2}$ is the systemic half-life of the drug and $C_{1, a v e, s s}$ is the steady state plasma drug concentration upon constant rate continuous intravenous infusion of the drug. The ratio $\frac{k_{0}}{C_{1, \text { ave ss }}}$ is the drug clearance which is in turn related to the apparent volume of distribution via the half-life.

\subsection{Multi-Compartment Models}

It is generally believed that the compartment volumes of multi-compartment pharmacokinetic models bear no physiological significance. As we have seen, in the open one-compartment model the apparent volume of distribution is equal to the amount of drug in the system over the drug concentration in the plasma at all times because the system is at continuous substance distribution equilibrium. Since in multi-compartment mammillary pharmacokinetic models the plasma drug concentration is equal to the amount of drug in the central compartment over the volume of the central compartment, the drug's apparent volume of distribution associated with the central compartment must be related to the actual volume of the central compartment (Equation (4a)). Therefore, the compartment volume assessed by regression analysis methods of measured plasma drug concentration with time must be the physiologically drug-accessible volume of the central compartment.

In the simplest multi-compartment model, the two-compartment model, the volume of the central compartment $V_{1}$ can be determined by Equation (8) but the notion that $V_{d, 1}$ is equal to $V_{1}$ is fundamentally wrong. As distribution of 
drug in the peripheral compartment is not instantaneous, at zero time all the drug is in the central compartment and the extrapolated to time zero plasma drug concentration $\left(C_{1,0}\right)$ must be related to the real volume of the central compartment.

$$
V_{1}=\frac{\operatorname{Dose}_{I V}}{C_{1,0}} \neq V_{d, 1}
$$

The accurate assessment of the volume of the central compartment $V_{1}$ from experimental measurements of drug concentration using regression techniques is of paramount importance not only because it is the actual physiological volume of the central compartment of the model but because it is the value that is also used to calculate $V_{d, 1}$ after computing the volume and the drug concentration of the peripheral compartment as a function of time. One of the problems of the model are the conditions of substance distributional equilibrium at which $V_{2}$ is currently being determined. Equation (9) indicates that $V_{2}$ can be calculated from $V_{1}$ and the intercompartmental drug transfer rate constants or from the mass of the solute in the two compartments when solute distribution rates between the two compartments are equal. The left-hand side of Equation (9) as derived from Equation (10) is stating that at equilibrium there is an equal mass transfer rate of solute between the two compartments in the system.

$$
\begin{gathered}
\frac{k_{21}}{k_{12}}=\frac{x_{1}}{x_{2}}=\frac{V_{1}}{V_{2}} \\
k_{12} \cdot x_{1}-k_{21} \cdot x_{2}=0
\end{gathered}
$$

The caveat of the method is that drug distribution equilibrium is decided from Fick's law based on conditions of equal drug concentration in the two compartments. Under these conditions the ratio of the intercompartmental transfer rate constant equals the volume ratio of the two phases. In other words, the method is accurate only for drugs that have a tissue-plasma partition coefficient approximately equal to one with the kinetically different compartments of the model being compositionally very similar.

In our recent article, we have calculated $V_{d, 1}$ of sisomicin after multiple intermittent intravenous infusions in a two-compartment model using Equation (4) [7]. As the concept of the steady-state plasma drug concentration is unrelated to the apparent volume of distribution, the apparent volume of distribution of a drug can be very carefully assessed after administration of single or multiple doses from drug concentrations that are measured exactly at the time at which the distribution rates of drug into the two compartments are equal. We must recognize that in the more realistic open two-compartment model (Figure 1, right) distributional equilibrium is not a lasting one. We would like to call this special event, where the non-steady state rates of drug distribution in the two compartments are balanced, a momentary equilibrium. This is true for all routes of drug administration except for the constant rate continuous intravenous infusion where intercompartmental drug distribution reaches a long-lasting equili- 
brium when the plasma drug concentration reaches steady state conditions. The time of momentary distributional equilibrium $\left(t_{e q}\right)$ was determined as a function of time and dose number from Equation (10). It was estimated that solute distribution between the two compartments reaches equilibrium at the same time the peripheral compartment peak concentration is observed, 27.7 -to-18.3 minutes after infusion has stopped, depending on the infusion number [7]. Keep in mind that with repetitive drug administration the $x_{s}$ on Equation (4), being the amount of drug in the system and not the drug dose, is not a constant quantity. The drug was administered every 180 minutes with a brief infusion of 30 minutes. The value of the $V_{d, 1}$ is calculated from the amount of drug in the body and the concentration in the central compartment $\left(C_{1, e q}\right)$ at $t_{e q}$, as a function of dose number, to be equal to $12.16 \mathrm{~L}$ (Table 1).

The volumes of the central and peripheral compartments as calculated by the original authors of the work were $5.17 \mathrm{~L}$ and $6.61 \mathrm{~L}$ [8]. These volumes represent the physiological volumes of extracellular fluid compartments that receive the drug at different kinetic rate. As with other aminoglycosides, total exclusion of the drug from intracellular fluids is most plausible considering the highly polar and hydrophilic nature of sisomicin. Also, the cationic nature of these molecules at physiological $\mathrm{pH}$ could be the reason for a delayed diffusion through the capillary of the endothelial cells, hence the kinetically different but compositionally similar peripheral compartment of extracellular fluid. Alternatively, the peripheral compartment could be an interstitial fluid phase of slightly higher drug affinity, where steric hindrance and binding to the negatively charged components of the extracellular matrix retards drug movement. In any event, our analysis suggests that classical pharmacokinetic modeling is able to provide an accurate description of the drug concentration with time for hydrophilic drugs that have access only to intravascular and interstitial fluids. The explicit solutions to the two-compartment model as a function of real time provide estimates of drug concentration upon repeated dosing but their clinical relevance still remains to be seen as the kinetically different compartments may not persist after the first dose [7].

Ideally, solute transfer at the interface between two immiscible phases reaches steady state when $C_{2}=K_{2,1} \cdot C_{1}$. We can combine this thermodynamic relationship denoting equal chemical potential of solute in each phase with the steady state Equation (10) where the mass transfer rate of the solute between the two phases does not change with time.

$$
\frac{k_{12}}{k_{21}}=K_{2,1} \cdot \frac{V_{2}}{V_{1}}
$$

Combining Equation (2) with Equation (11) yields the familiar Riggs equation.

$$
V_{d, 1}=V_{1} \cdot\left(1+\frac{k_{12}}{k_{21}}\right)
$$


Table 1. Sisomicin's apparent volume of distribution associated with the central compartment was calculated from the mass of solute in the central and peripheral compartment at times of distributional equilibrium after repetitive intermittent intravenous infusions in a mammillary two-compartment model [7].

\begin{tabular}{lcccc}
\hline$n$ & $x_{1, e q}(\mathrm{mg})$ & $x_{2, e q}(\mathrm{mg})$ & $C_{1, e q}(\mathrm{mg} / \mathrm{L})$ & $V_{d, 1}(\mathrm{~L})$ \\
\hline 1 & 16.71 & 20.39 & 3.05 & 12.16 \\
2 & 23.37 & 28.51 & 4.27 & 12.16 \\
3 & 25.82 & 31.50 & 4.72 & 12.16 \\
4 & 26.71 & 32.59 & 4.88 & 12.16 \\
5 & 27.03 & 32.98 & 4.94 & 12.16 \\
6 & 27.15 & 33.12 & 4.96 & 12.16 \\
\hline
\end{tabular}

Equation (12) can be used to assess a substance apparent volume of distribution associated with the phase of the central compartment but only if the central compartment is a single phase. This was the case of Prazosin where although the drug appeared to be distributed in the whole total body water, the volume of the central compartment was roughly equal to the extracellular fluid volume. Using Equation (12), the apparent volume of Prazosin as determined from single IV bolus injection clinical data using a two-compartment open model was found to be $32.9 \pm 6.9 \mathrm{~L}$ [9]. Using Equation (12), the $V_{d, 1}$ of Sisomicin was calculated to be equal to $11.78 \mathrm{~L}$. The equilibrium time of intercompartmental drug distribution $t_{e q}$ is calculated from Equation (10) whereas the compartment volumes were estimated by regression analysis of measured plasma drug concentration with time, hence the small difference between the two calculated values of the apparent volume of distribution (Table 1).

Equation (11) contains two unknowns, the partition coefficient, $K_{2,1}$ and $V_{2}$. In order to calculate the volume of peripheral compartment(s) the "tissue-plasma" partition coefficient(s) must be determined with a different experiment unless if it is verified experimentally that the peripheral compartment is a single organ and a single phase. In that case, the apparent volume of distribution $V_{d, 1}$ determined from a measured plasma drug concentration and the amount of drug in the body at $t_{e q}$ using Equation (13), can be used to compute the organ-plasma partition coefficient from $V_{1}$ and the corresponding organ volume using Equation (2). One would expect the magnitude of $k_{12}$ to be much higher than $k_{21}$ for lipophilic drugs but as the mass transfer rates are affected by the actual compartment volumes the presence of another phase in the system cannot be ascertained merely by the difference in the relative magnitude of these two constants. On the other hand, an apparent volume of distribution greater than the total body water is a definite indication of the presence of another phase in the system.

An extensive literature search for lipophilic drug multi-compartment pharmacokinetic model data simulation indicated that most research articles, especially from population PK models, do not report the apparent volume of distri- 
bution. Furthermore, if the estimated volumes of the central and peripheral compartments are incorrect the intercompartmental clearances that these softwares calculate will also be inaccurate. The central compartment of lipophilic drugs is consistently reported to have a much higher than the extracellular fluid volume and frequently higher than total body water. It is possible that for these drugs the central compartment of a multi-compartment mammillary model contains two (or more) kinetically indistinguishable phases instead of a single phase. The plasma is part of phase $a$ that is in contact with phase $b$ in the central compartment and therefore measured plasma drug concentrations are affected by the drug distribution in phase $b$ within the central compartment. The apparent volume of distribution of the drug in phase $a$ is equal to the volume of phase $a$ and the sums of the products of the volume of phase $b$ and all other phases with the corresponding partition coefficients (Equation (13)). Without the tissue-plasma partition coefficient, it will not be possible to determine neither the actual volume of the central compartment from the plasma drug concentration at time zero nor their apparent volume of distribution for these drugs. However, measured plasma drug concentrations do reflect the drug that is present in both phases of the central compartment and thus, the drug's apparent volume of distribution can still be determined with Equation (4) as long as the total amount of drug in the body and the plasma drug concentration are calculated and determined, respectively, at times of momentary distribution equilibrium, $t_{e q}$ (Equation (14)). It is important to say, that the amount of drug in the peripheral compartment can be calculated without knowing the volume of the peripheral compartment(s) [7] [9].

$$
\begin{gathered}
V_{d, 1 a}=V_{1 a}+V_{1 b} \cdot K_{b, a}+\sum_{i=2}^{\infty} V_{i} \cdot K_{i, 1 a} \\
V_{d, 1, a}=\frac{x_{s, e q}}{C_{1, a, e q}}
\end{gathered}
$$

Being able to explain multi-compartment pharmacokinetic models comes with an additional advantage, that is, comparison simulations of drug concentration among various compartment models. We have shown that the apparent volume of distribution of a drug associated with the central phase in a two-compartment model is the same as the drug's apparent volume of distribution with respect to plasma. Its value as determined from the two-compartment model can be used to carry out simulations of drug concentration with time in the one-compartment model. Simulations of this kind can assess if dosage calculations of a two-compartment model drug can be approximated with the straightforward analytical solutions of the one-compartment model which can easily be employed in a clinical setting. That was the case with Prazosin where although the drug follows a two-compartment model, intermittent infusion dosage calculations can be safely handled using the much simpler one-compartment model [9]. Similarly, administering repetitive Sisomicin intravenous doses that were calculated with analytical solutions of the one-compartment model, yet with pharmacokinetic 
parameters estimated from a two-compartment model resulted in an average steady-state plasma concentration only $26 \%$ higher than the one calculated from equations of the two-compartment model [7].

Before proceeding a word about Riggs work is appropriate [10]. In his excellent chapter "Kinetics of transfer between compartments" he defines a term, $(V \text { dist })_{B_{A}}=\frac{Q_{B, e q}}{C_{A, e q}}$, which he calls the volume of distribution in compartment B with reference to its concentration in compartment $\mathrm{A}$ at equilibrium, and then he uses the partition coefficient to produce Equation (12). The term $(V \text { dist })_{B_{A}}$ is not equivalent to the volume of distribution of solute in compartment $B$ and it is not the apparent volume of distribution with respect to compartment B. As a reminder, the apparent volume of distribution with respect to compartment $\mathrm{B}$ is $V_{d, B}=\frac{Q_{\text {total }, e q}}{C_{B, e q}}=\frac{Q_{\text {total }, e q}}{K_{2,1} \cdot C_{A, e q}}$ (Equation (5)). Riggs does not explain the physical meaning of this term, and he does not calculate the volume of compartment B or the value of the partition coefficient. He doesn't have to because his work is all about the actual volume of distribution of creatinine in the body from its concentration in the plasma and not the apparent volume of distribution as we have defined it here. From our analysis herein and elsewhere, the term $(V \text { dist })_{B_{A}}$ is the volume of the central compartment that is needed to dissolve the amount of solute that is present in the peripheral compartment when the two compartments/phases are in conduct and at equilibrium [5]. Because the concept of the apparent volume of distribution was never defined, and because calculations of the volume of distribution are always carried out using the micro rate constants, the middle part of (15) gradually became simply $V_{A}+V_{B}$, as the partition coefficient of the two reference regions of identical solubility in the two compartments is always equal to one [11] [12].

$$
(\text { V dist })=V_{A}+(V \text { dist })_{B_{A}}=V_{A}+V_{B} \cdot R_{B / A}=V_{A} \cdot\left(1+\frac{k_{A B}}{k_{B A}}\right)
$$

For Riggs, Equation (12) was perfect to calculate the actual volume of distribution of creatinine as its size is about the volume of total body water. However, investigators that are studying other lipophilic multi-compartment model drugs that exhibited an estimated apparent volume of distribution much larger than the total body fluids, without considering the possibility of having different phases within compartments and without knowing the concept of the apparent volume of distribution as we have defined it herein, they have trouble explaining the calculated compartment volumes and their physical significance.

There are a number of other methods to estimate the apparent volume of distribution with respect to the plasma or the central compartment such as, the extrapolation method $\left(V_{d, \text { extrap }}\right)$, the area $\left(V_{d, \text { area }}\right)$ or clearance method $\left(V_{d, \beta}\right)$ and the steady-state volume of distribution $\left(V_{d, s s}\right)$ [13]. The problems of estimating the volume of distribution by the extrapolation method were already discussed extensively by Riggs. Also, Gibaldi and coworkers have already shown 
that the $V_{d, \text { area }}$ is identical to the $V_{d, \beta}$ [14]. As far as the $V_{d, s s}$ is concerned there appears to be two equations that can be used to calculate its value. The first one is the Riggs equation that we have just discussed for the two-compartment model. The second equation was derived by Wagner for the intravenous bolus injection route of administration where the volume of distribution can be determined from the exponents and coefficients of the explicit solution to drug concentration of the central compartment in multi-compartment pharmacokinetic models [10] [15]. In particular, Wagner has shown that these equations yield $V_{d, s s}$ values very similar to those determined by Riggs equation [15] [16]. Wagner has used a very different approach than Riggs but essentially the two forms are the same equation (Appendix).

In physiologically-based pharmacokinetic modeling (PBPK) predictions of the apparent volume of distribution are carried out using the familiar equation,

$$
V_{d, s s}=V_{p}+\sum_{i=1}^{n} V_{t, i} \cdot K_{t, i, p}
$$

The $V_{d, s s}, V_{p}, V_{t}$ and $K_{t, p}$ are the apparent volume of distribution, the volume of plasma, the volume of tissue and the tissue-plasma partition coefficient, respectively. The term steady state (apparent) volume of distribution must be used with caution. The conditions necessary to determine a drug's apparent volume of distribution in multi-compartment models are equal mass transfer rates between compartments and not steady-state plasma drug concentration. The only mode of administration that ensures a near zero fluctuation in steady state plasma drug concentration and hence long lasting equal intercompartmental mass transfer rates, is the constant rate continuous intravenous infusion [17]. Clearly, in PBPK modeling every tissue compartment constitutes a different phase in contact with the central circulation but the partition coefficient is multiplied by the tissue volume instead of the tissue phase volume. The accuracy of the model could be improved using the extracellular fluid instead of the plasma volume and by adjusting the tissue volume without the interstitial fluid that is exchanged with the central phase. Although the apparent volume of distribution is not used in PBPK modeling, the tissue-plasma partition coefficients are the key parameters for predicting the time course of plasma drug concentration in PBPK simulations. There are various methods to continuously monitor in vivo plasma and tissue drug concentrations but the partition coefficient must be determined at the time of momentary distribution equilibrium $\left(t_{e q}\right)$, and not when the ratio of the drug concentration in the two tissues stays constant ( $V_{d}$ vs $\left.V_{\beta}\right)$ [7].

One of the advantages of the apparent volume of distribution is that it can be used to determine the partition coefficient in any biphasic system e.g., suspensions, emulsions, liposomes, cultured cells, tissue homogenates or whole tissue by measuring the drug concentration only in one of the phases in a series of in vitro equilibrium titration experiments [5]. The experimentally determined values of $K_{t, 1, p}$ can be used in PBPK modeling to provide a more accurate simulation of in vivo drug concentration in the tissues using the arterial drug concentration and 
the tissue blood flow. The drug's in vitro apparent volume of distribution with respect to the tissue, is $V_{d, t}=\frac{V_{d, 1}}{K_{t, 1}}$ and the in vitro phase drug concentration can be calculated from $C_{t}=\frac{x_{s}}{V_{d, t}}$.

\section{Conclusion}

The classical pharmacokinetic compartment models were never explained because the apparent volume of distribution was never understood and never defined scientifically. The difference between the two terms, volume of distribution as opposed to the apparent volume of distribution, has now become clear. In the one-compartment model, the apparent volume of distribution is always equal to the product of drug clearance with the elimination rate constant. It can be used to compute the drug amount in the body from measured plasma drug concentration, at all times. In the multi-compartment models, the apparent volume of distribution is directly related to the drug's actual volume of distribution in the body and it can relate the drug amount in the body to the measured plasma drug concentration only at times of drug distribution equilibrium between compartments. Its value is still reflective of the extent of drug distribution in the body. Apparent volumes of distribution less than total extracellular fluid volume, suggest presence of single phase whereas apparent volumes of distribution larger than total body water imply presence of multiple drug partition phases in single or multi-compartment kinetic models. According to our analysis, the classical pharmacokinetic compartmental modeling can provide accurate and full description of disposition kinetics of hydrophilic drugs but it cannot provide an estimate of the apparent volume of distribution for lipophilic drugs that do not follow one-compartment model and their estimated central compartment volume is larger than the total body fluid volume. The simulations in Figure 3, bottom panel, suggested that potential differences in the apparent volume of distribution of healthy individuals can be minimized after normalizing the values by the total body weight. Individual populations with disease states and conditions that can cause abnormal body fluid distribution and volume must be handled separately as their apparent volume of distribution is different from the average healthy population. It becomes more and more evident, that for accurate pharmacokinetic parameter estimation, data simulation should be conducted by combined nonlinear mixed-effect and structural models. The field of pharmacokinetics has hitherto two kinds of incompatible models: the classical pharmacokinetic modeling that can only study kinetically different compartments and the PBPK modeling where every compartment is a different phase and is unable to simulate compositionally similar, kinetically different, compartments. These two models cannot be unified into one, but the classical pharmacokinetic compartment model can be treated as a multiphasic compartment model to provide accurate estimates of the apparent volume of distribution of a drug from the vo- 
lume of kinetically different compartments, instead of plasma, as the central phase, and the volumes of different phase compartments that are in contact with the central phase.

\section{Conflicts of Interest}

The author declares no conflicts of interest regarding the publication of this paper.

\section{References}

[1] Dominguez, R. (1934) Studies of Renal Excretion of Creatinine. II. Volume of Distribution. Proceedings of the Society for Experimental Biology and Medicine, 31, 1146-1149. https://doi.org/10.3181/00379727-31-7476C

[2] Wagner, J.G. (1981) History of Pharmacokinetics. Pharmacology \& Therapeutics, 12, 537-562. https://doi.org/10.1016/0163-7258(81)90097-8

[3] Dominguez, R. (1934) Studies of Renal Excretion of Creatinine. III. Utilization Constant. Proceedings of the Society for Experimental Biology and Medicine, 31, 1150-1154. https://doi.org/10.3181/00379727-31-7477C

[4] Winkler, A.W. and Smith, P.K. (1938) The Apparent Volume of Distribution of Potassium Injected Intravenously. Journal of Biological Chemistry, 124, 589-598. https://doi.org/10.1016/S0021-9258(18)74020-9

[5] Savva, M. (2021) On the Origin of the Apparent Volume of Distribution and Its Significance in Solvent Extraction Methods. Journal of Organic Chemistry and Chemical Sciences, 1, 101. https://doi.org/10.17303/jocs.2021.1.101

[6] Savva, M. (2019) Mixture Problems in Pharmaceutical Calculations. In: Pharmaceutical Calculations. A Conceptual Approach, Springer International Publishing, Berlin, 113-155. https://doi.org/10.1007/978-3-030-20335-1_6

[7] Savva, M. (2022) Real-Time Analytical Solutions as Series Formulas and Heaviside off/on Switch Functions for Multiple Intermittent Intravenous Infusions in the One- and Two-Compartment Models. Journal of Biosciences and Medicines. (In Press)

[8] Pechere, J.C., Pechere, M.M. and Dugal, R. (1976) Clinical Pharmacokinetics of Sisomicin: Two-Compartment Model Analysis of Serum Data after IV and IM Administration. European Journal of Clinical Pharmacology, 10, 251-256. https://doi.org/10.1007/BF00558337

[9] Savva, M. (2022) A Reevaluation of Prazosin Pharmacokinetics in a Two-Compartment Model: The Apparent Volume of Distribution and Comparative Simulations in the One-Compartment Model. Journal of Biosciences and Medicines. (In Press)

[10] Riggs, D.S. (1963) Transfer of Substances between Biological Compartments. General Kinetics. In: The Mathematical Approach to Physiological Problems, Williams and Wilkins, Baltimore, 193-217.

[11] Benet, L.Z. and Ronfeld, R.A. (1969) Volume Terms in Pharmacokinetics. Journal of Pharmaceutical Sciences, 58, 639-641. https://doi.org/10.1002/jps.2600580534

[12] Nagashima, R., Levy, G. and O’Reilly, R.A. (1968) Comparative Pharmacokinetics of Coumarin Anticoagulants IV. Application of a Three-Compartmental Model to the Analysis of the Dose-Dependent Kinetics of Bishydroxycoumarin Elimination. Journal of Pharmaceutical Sciences, 57, 1888-1895.

https://doi.org/10.1002/jps.2600571113 
[13] Riegelman, S., Loo, J. and Rowland, M. (1968) Concept of a Volume of Distribution and Possible Errors in Evaluation of This Parameter. Journal of Pharmaceutical Sciences, 57, 128-133. https://doi.org/10.1002/jps.2600570125

[14] Gibaldi, M., Nagashima, R. and Levy, G. (1969) Relationship between Drug Concentration in Plasma or Serum and Amount of Drug in the Body. Journal of Pharmaceutical Sciences, 58, 193-197. https://doi.org/10.1002/jps.2600580210

[15] Wagner, J.G. (1976) Linear Pharmacokinetic Equations Allowing Direct Calculation of Many Needed Pharmacokinetic Parameters from the Coefficients and Exponents of Polyexponential Equations which Have Been Fitted to Data. Journal of Pharmacokinetics and Biopharmaceutics, 4, 443-467. https://doi.org/10.1007/BF01062831

[16] Benet, L.Z. (1979) Noncompartmental Determination of the Steady-State Volume of Distribution. Journal of Pharmaceutical Sciences, 68, 1071-1074.

https://doi.org/10.1002/jps.2600680845

[17] Gibaldi, M. (1969) Effect of Mode of Administration on Drug Distribution in a Two-Compartment Open System. Journal of Pharmaceutical Sciences, 58, 327-331. https://doi.org/10.1002/jps.2600580308 


\section{Appendix}

Equation for Intravenous bolus injection in a two-compartment mammillary model,

$$
V_{d, s s}=D_{i v} \cdot \frac{\sum_{i=1}^{n} \frac{A_{i}}{\lambda_{i}^{2}}}{\left(\sum_{i=1}^{n} \frac{A_{i}}{\lambda_{i}}\right)^{2}}
$$

For a two-compartment mammillary model,

$$
C_{p}(t)=C_{1}(t)=A_{1} \cdot \mathrm{e}^{-\lambda_{1} \cdot t}+A_{2} \cdot \mathrm{e}^{-\lambda_{2} \cdot t}
$$

Thus,

$$
V_{d, s s}=D_{i v} \cdot \frac{\left(\frac{A_{1}}{\lambda_{1}^{2}}+\frac{A_{2}}{\lambda_{2}^{2}}\right)}{\left(\frac{A_{1}}{\lambda_{1}}+\frac{A_{2}}{\lambda_{2}}\right)^{2}}=D_{i v} \cdot \frac{\frac{\left(\lambda_{2}^{2} \cdot A_{1}+\lambda_{1}^{2} \cdot A_{2}\right)}{\lambda_{1}^{2} \cdot \lambda_{2}^{2}}}{\frac{\left(\lambda_{2} \cdot A_{1}+\lambda_{1} \cdot A_{2}\right)^{2}}{\lambda_{1}^{2} \cdot \lambda_{2}^{2}}}
$$

But, $A_{1}=\frac{D_{i v}}{V_{1}} \cdot \frac{\left(k_{21}-\lambda_{1}\right)}{\left(\lambda_{2}-\lambda_{1}\right)} ; \quad A_{2}=\frac{D_{i v}}{V_{1}} \cdot \frac{\left(\lambda_{2}-k_{21}\right)}{\left(\lambda_{2}-\lambda_{1}\right)}$;

Thus,

$$
\begin{aligned}
& V_{d, s s}=\frac{D_{i v} \cdot \frac{D_{i v}}{V_{1}} \cdot\left(\frac{\lambda_{2}^{2} \cdot\left(k_{21}-\lambda_{1}\right)+\lambda_{1}^{2} \cdot\left(\lambda_{2}-k_{21}\right)}{\left(\lambda_{2}-\lambda_{1}\right)}\right)}{\frac{D_{i v}^{2}}{V_{1}^{2} \cdot\left(\lambda_{2}-\lambda_{1}\right)^{2}} \cdot\left(\lambda_{2} \cdot\left(k_{21}-\lambda_{1}\right)+\lambda_{1} \cdot\left(\lambda_{2}-k_{21}\right)\right)^{2}} \\
& =\frac{V_{1} \cdot\left(\lambda_{2}-\lambda_{1}\right) \cdot\left(\lambda_{2}^{2} \cdot\left(k_{21}-\lambda_{1}\right)+\lambda_{1}^{2} \cdot\left(\lambda_{2}-k_{21}\right)\right)}{\left(\lambda_{2} \cdot k_{21}-\lambda_{1} \cdot k_{21}\right)^{2}} \\
& =\frac{V_{1} \cdot\left(\lambda_{2}-\lambda_{1}\right) \cdot\left(\lambda_{2}^{2} \cdot k_{21}-\lambda_{1} \cdot \lambda_{2}^{2}+\lambda_{1}^{2} \cdot \lambda_{2}-\lambda_{1}^{2} \cdot k_{21}\right)}{k_{21}^{2} \cdot\left(\lambda_{2}-\lambda_{1}\right)^{2}} \\
& =\frac{V_{1} \cdot\left(k_{21} \cdot\left(\lambda_{2}^{2}-\lambda_{1}^{2}\right)+\lambda_{1} \cdot \lambda_{2} \cdot\left(\lambda_{2}-\lambda_{1}\right)\right)}{k_{21}^{2} \cdot\left(\lambda_{2}-\lambda_{1}\right)} \\
& =\frac{V_{1} \cdot\left(k_{21} \cdot\left(\lambda_{1}+\lambda_{2}\right)-\lambda_{1} \cdot \lambda_{2}\right)}{k_{21}^{2}}
\end{aligned}
$$

Let, $\lambda_{1} \cdot \lambda_{2}=k_{10} \cdot k_{21}$ and $\lambda_{1}+\lambda_{2}=k_{12}+k_{10}+k_{21}$

$$
\Rightarrow V_{d, s s}=V_{1} \cdot\left(1+\frac{k_{12}}{k_{21}}\right)
$$

